\title{
Extraction of Connected Region Boundary in Multidimensional Images
}

\author{
David Coeurjolly ${ }^{1}$, Bertrand Kerautret ${ }^{2}$, Jacques-Olivier Lachaud ${ }^{3}$ \\ ${ }^{1}$ CNRS, LIRIS UMR5205, Université de Lyon \\ ${ }^{2}$ LORIA, Université de Lorraine \\ ${ }^{3}$ LAMA, Université de Savoie
}

Communicated by Bertrand Kerautret

Demo edited by Bertrand Kerautret

\begin{abstract}
This paper presents an algorithm to extract the boundary of a connected region(s) using classical topology definitions. From a given adjacency definition, the proposed method is able to extract the boundary of an object in a generic way, independently of the dimension of the digital space.

\section{Source Code}

The implementation of the algorithm is available through the DGtal library ${ }^{1}$. The source code and the demonstration are based on a special version of DGTal containing no external dependencies. They are both available on the IPOL web page of this article ${ }^{2}$.
\end{abstract}

\section{Supplementary Material}

The DGtalTools ${ }^{3}$ project gives several additional tools exploiting the proposed algorithms. These tools are defined to process both 2D and 3D images.

Keywords: Nd connected components, discrete geometry, topology

\section{Introduction}

The aim of this work is to present an algorithm which extracts the boundary of connected region(s) using classical topology definitions. It can be useful in particular for algorithms which need a discrete contour as input. Such contours can be extracted from grayscale images like the ones displayed in Figure 1.

These digital contours are level set contours in the displayed grey-level image, specified by a threshold parameter (i.e. the level set), a reference point and a maximal distance. In order to describe

\footnotetext{
${ }^{1}$ DGtal: Digital Geometry tools and algorithms library, http://libdgtal.org

${ }^{2}$ https://doi.org/10.5201/ipol.2014.74

${ }^{3}$ https://github.com/DGtal-team/DGtalTools
} 


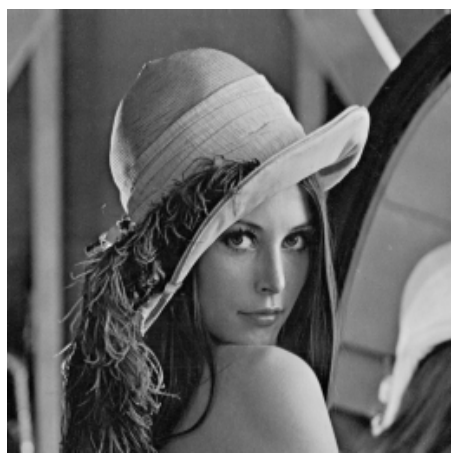

(a)

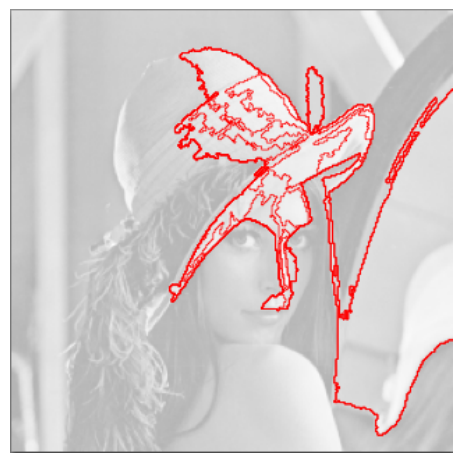

(b)

Figure 1: Example of contour extraction (b) from source image (a).

in a generic way the object on which the contour is extracted, the user must specify a predicate on volume elements which specifies whether or not a given element belongs to the region(s) of interest. This information is sufficient to extract the interpixel boundaries of this or these region(s). The boundaries are extracted by tracking along the frontier between the region and its complementary. The user may choose between two adjacency definitions for object(s): the interior and the exterior adjacency. The extracted set of connected surface elements satisfies the choice of adjacency. In dimension 2, the surface elements can be ordered to form contour(s) composed of 4-connected points in the half-integer plane. Predicates on volume elements can be very simple such as the example described in Figure 1: the predicate is just a composition of thresholds on the image values.

Complexity note: extracting all the contours for a given predicate takes $O(M N)$ calls to the predicate, if $M$ and $N$ are respectively the width and height of the image. In arbitrary dimension, the computational cost is proportional to the size of the predicate domain (number of grid points). Extracting a single contour given a starting boundary element takes $O(C)$ calls to the predicate, if $C$ is the number of points of this contour.

Implementation note: although the examples and applications are given in $2 \mathrm{D}$ and $3 \mathrm{D}$, the surface extraction algorithm is presented and implemented for arbitrary dimension. The applications of Section 3 can be reproduced from the demo version given IPOL web page of this article ${ }^{4}$.

\section{Algorithm}

\subsection{Digital Surfaces for Boundary Extraction}

Different definitions of digital surfaces can be found in the digital topology domain (refer to the paper by Kong et al. [2] for a survey). A first group of approaches was introduced by Rosenfeld in the 70s $[5,6]$ which consists in defining a digital surface as a subset $S$ of $\mathbb{Z}^{n}$ with the property to have $\mathbb{Z}^{n} \backslash S$ composed of two $\alpha$-connected (with for instance $\alpha=4$ or $\alpha=8$ for the square pixels of an 2D image) components and $S$ is thin (i.e. if any point of $\mathrm{S}$ is removed, the preceding property does not hold). Even if such a definition can be used in 2D, it appears more difficult to exploit in higher dimension. A second type of definition considers surfaces as $n-1$ dimensional cubical complexes [3]. This representation is convenient to describe the object but is not adapted to process geometric information of the object boundary. To extract the boundary of a digital surface, we shall use another definition which relies on the set of $n$-1-cells with some specific adjacency (in the same

\footnotetext{
${ }^{4}$ https://doi.org/10.5201/ipol.2014.74
} 
spirit as Herman [1] or Udupa [7]). Note that we consider here only the implementation for regular grids.

Digital surface as a set of $n-1$-cells. Formally, the elements of a digital space $\mathbb{Z}^{n}$ are called spels (and often pixels in 2D and voxels in 3D). A surfel is a couple $(u, v)$ of face adjacent spels. A digital surface is a set of surfels. A spel is thus a $n$-cell in a cellular grid decomposition of the space, while a surfel is clearly some oriented $n$-1-cell which is incident to the two $n$-cells (see figure 2).

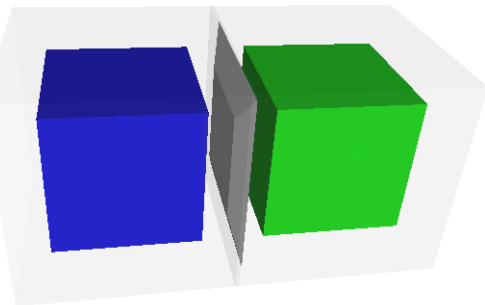

Figure 2: Illustration of a surfel $(n-1$-cell) with its two incident spels ( $n$-cells) in dimension 3.

From the implementation point of view, the set of $n$-1-cells can be obtained with an incidence relation from the given spels. In order to be able to extract object boundaries in a consistent way, we also consider cells with a specific orientation (positive or negative). By convention, spels lying in the interior of the object of interest are given a positive orientation. In the DGtal library framework, the orientation can be specified in the KSpace class (see user documentation ${ }^{5}$ ):

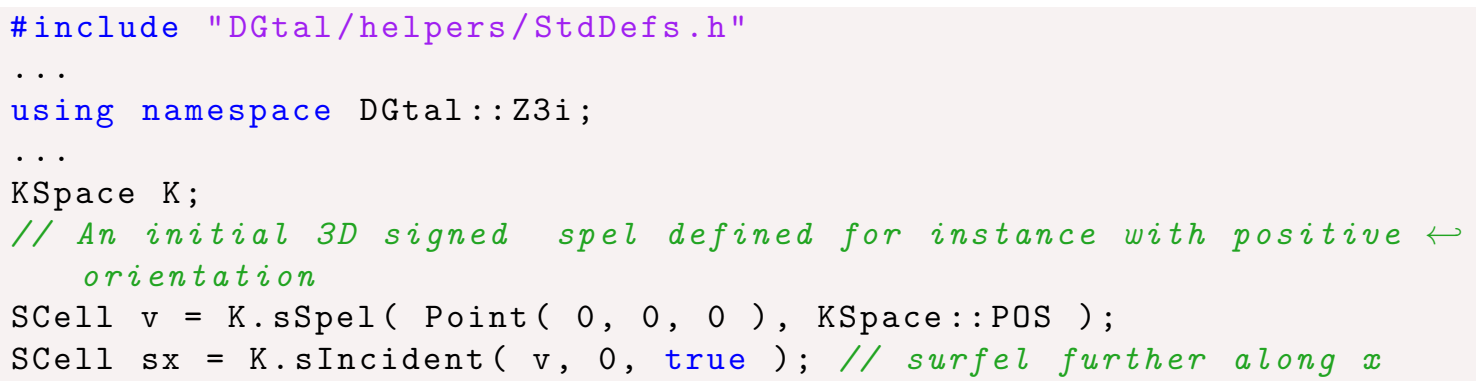

We can now obtain the digital surface that lies in the boundary of some digital shape $S \subset \mathbb{Z}^{n}$ as the set of oriented surfels between spels of $S$ and spels not belonging in $S$. Algebraically, $S$ is the formal sum of its positively oriented spels, and its boundary is obtained by applying the boundary operator on $S$. Figure 3 illustrates the linear boundary operator applied on the set of spels of the object. By linearity, the operator is applicable spel by spel and when opposite cells appear they cancel each other (see case $(\mathrm{c}, \mathrm{d})$ and $(\mathrm{h}, \mathrm{i})$ ).

Algorithm 1 exploits this definition to extract, given an input shape, the set of surfels that constitutes the shape boundary independently of its dimension. A $n \mathrm{D}$ digital Khalimsky space (the class KhalimskySpaceND ${ }^{6}$ in the DGtal library) is used to represent the cubical grid complex, whose oriented cells are defined as an array of integers (see the paper by Lachaud [4] for more details). In particular, the method uSpel (Point p) creates a cell of maximal dimension from a point with coordinates in $\mathbb{Z}^{n}$. Inversely, the coordinate of a Cell can be recovered from a Cell with the method uCoords (Cell c).

\footnotetext{
${ }^{5}$ http://libdgtal.org/doc/nightly/dgtal_cellular_topology.html

${ }^{6}$ http://libdgtal.org/doc/nightly/classDGtal_1_1KhalimskySpaceND.html
} 


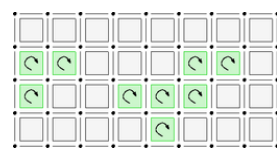

(a)

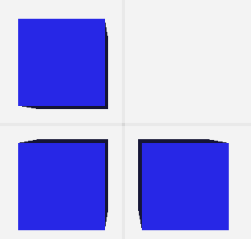

(f)

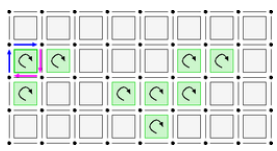

(b)

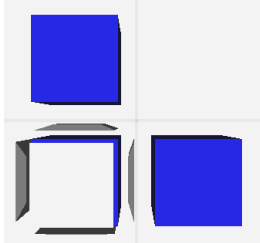

(g)

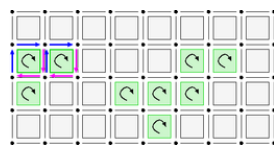

(c)

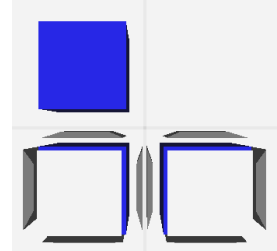

(h)

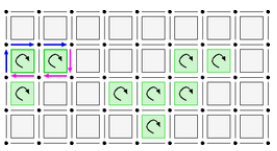

(d)

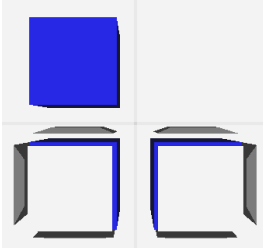

(i)

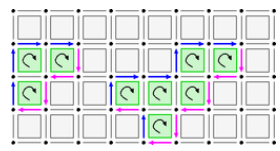

(e)

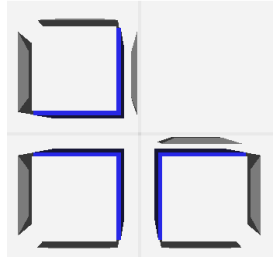

(j)

Figure 3: Application of the boundary operator to compute the boundary of a digital shape $S$, defined either as a 2D set of pixels (images (a-e)) or a 3D set of voxels (images (f-i)).

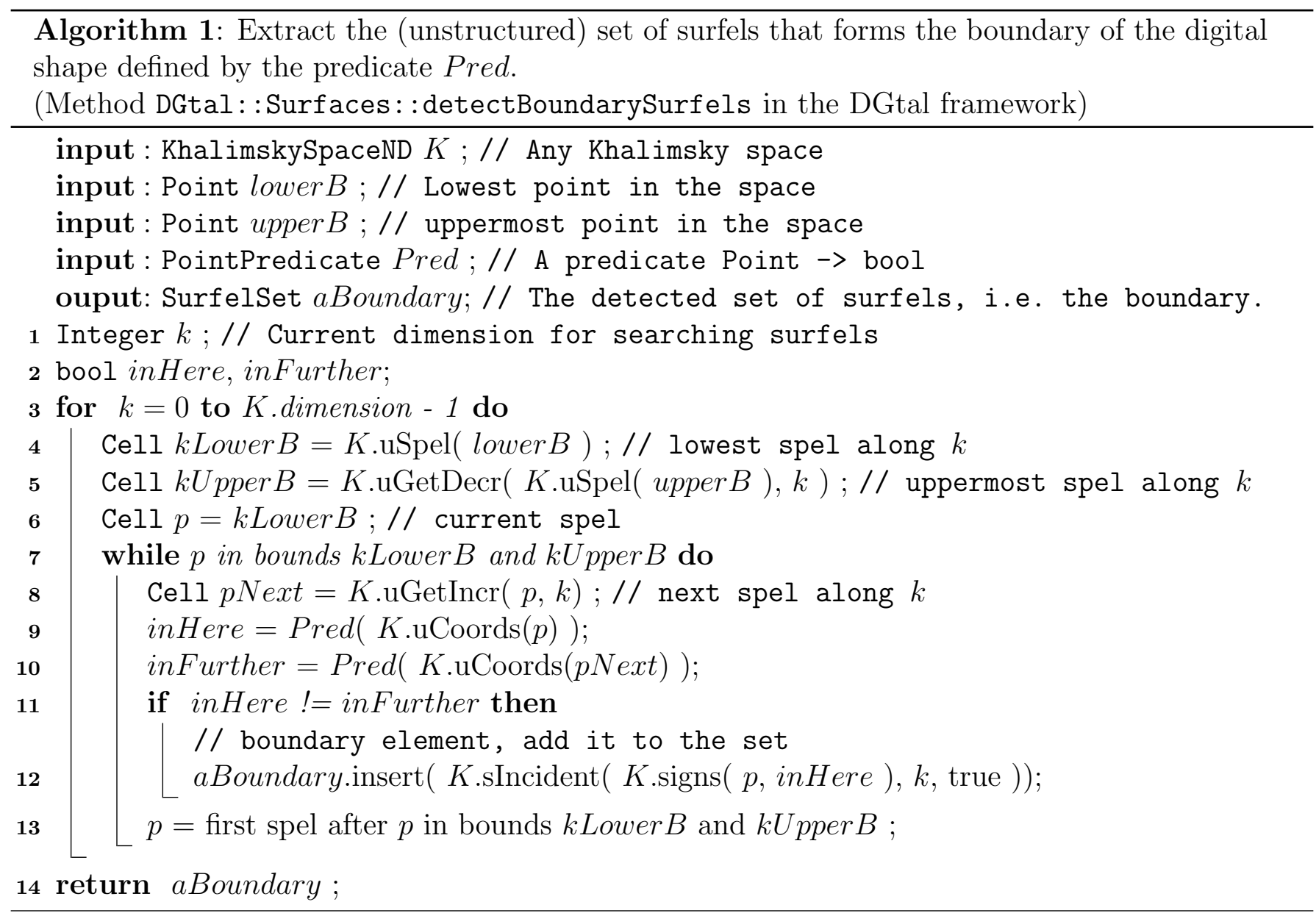


The boundary operator algorithm is given by the method DGtal::Surfaces<TKSpace $>$ : detectBoundarySurfels from the directory DGtal/topology/helpers. Images (a,f) of Figure 4 illustrate the result of this method applied on a set of pixels (a) and on a set of voxels (f) by calling the same method detectBoundarySurfels.

Once the digital set of surfels is defined, the relation between surfels needs to be determined to transform the digital surface into a graph.

Digital surface as a graph: adding adjacencies between surfels To apply this transformation we have to connect surfels that share $n-2$-cells. The resulting adjacency relation are called bel adjacencies in the terminology of Herman [1], Udupa [7] and others. Generally an $n-2$-cell is shared by two $n$-1-cells, except in "cross configuration" which are illustrated in the figure on the right.

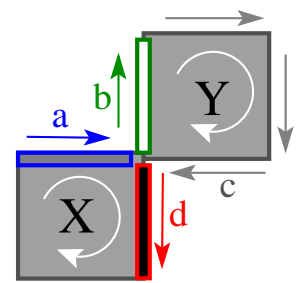

The interior bel adjacency makes the choice to connect $a$ to $d$ and $c$ to $b$ while the exterior bel adjacency connects $a$ to $b$ and $c$ to $d$. This choice has to be made along each possible pair of directions when going $n \mathrm{D}$. In DGtal, it is encoded through the class SurfelAdjacency. Images (b,g) in Figure 4 illustrate such possible choices for a given surfel in 2D and 3D. More precisely the green (resp. red) surfel associated to the choice of exterior (resp. interior) adjacency is obtained as follows:

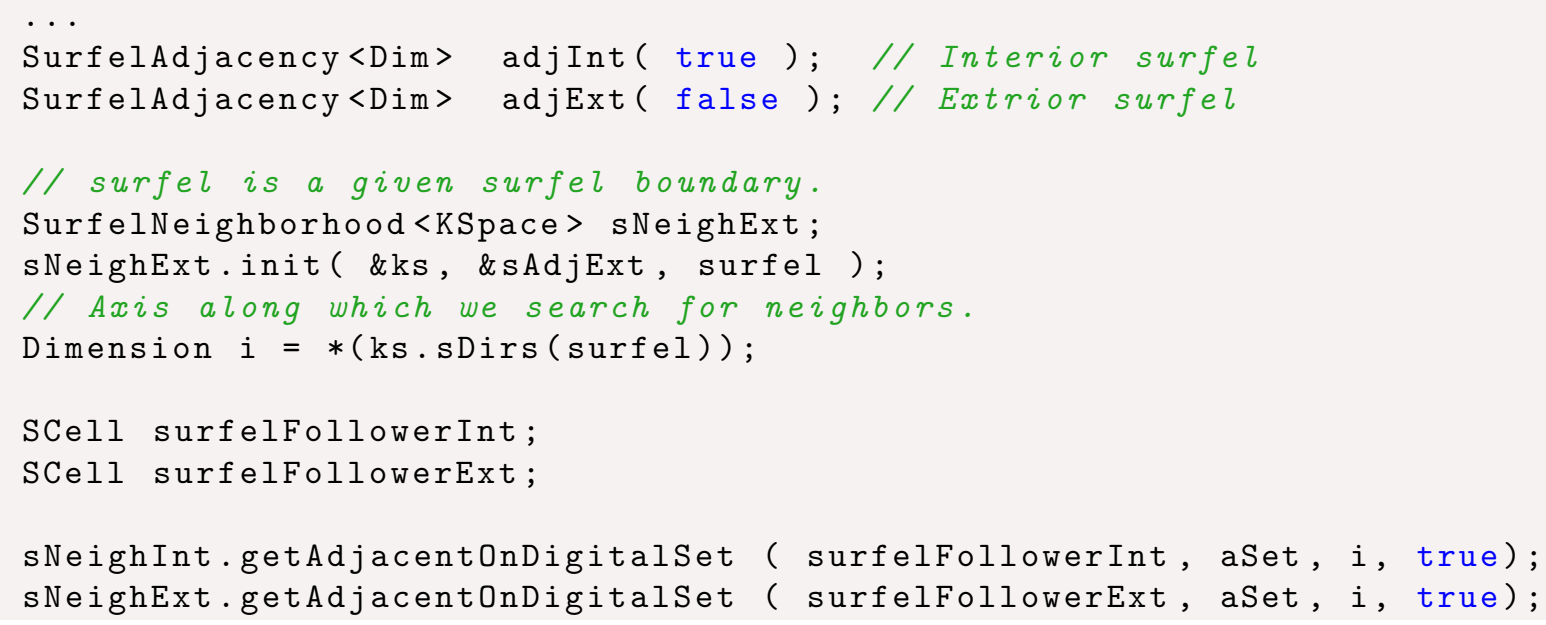

A more "classical topology" way of interpreting adjacencies is to consider an $\epsilon$-offset to the set of cells (see Figure 5). In 2D, an outward $\epsilon$-offset to the boundary cells (or equivalently, the boundary of the Minkowski sum of the set of spels and an $\epsilon$-ball) defines a 1-dimensional surface whose topology corresponds to the exterior adjacency. Similarly, an inward $\epsilon$-offset to the boundary cells defines a 1-dimensional surface whose topology corresponds to the interior adjacency. Unfortunately, this is no more true in 3D. Indeed, an outward $\epsilon$-offset in the great diagonal configuration (two diagonally opposite spels in $2 \times 2 \times 2$ cube) connects the surface cells, but they are not connected by the digital exterior adjacencies. However, the inward $\epsilon$-offset to the set of cells still defines the interior adjacency.

\subsection{Tracking Digital Surfaces}

Once the surfels separating interior spels from exterior spels have been extracted by Algorithm 1, the final step of tracking can be applied. We propose Algorithm 2 which is based on the more generic method getAdjacentOnPointPredicate, which only requires a predicate on a digital point. A digital set $S$ is then simply defined as the predicate returning true on points belonging to $S$. This algorithm is independent of the chosen dimension. This method can be used indifferently for an open 


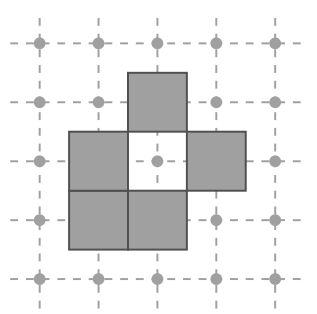

(a)

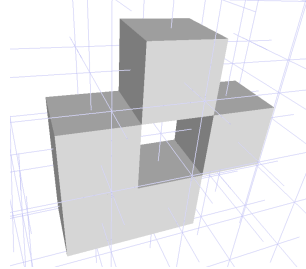

(f)

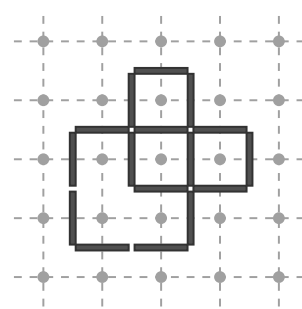

(b)

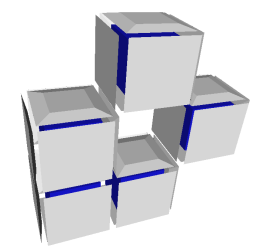

$(\mathrm{g})$

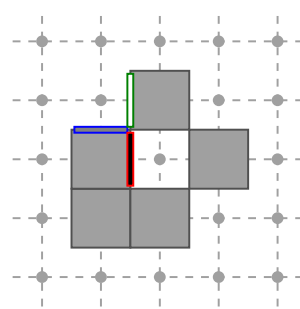

(c)

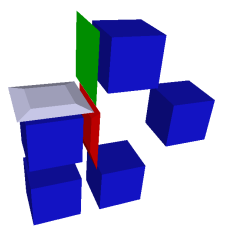

(c)

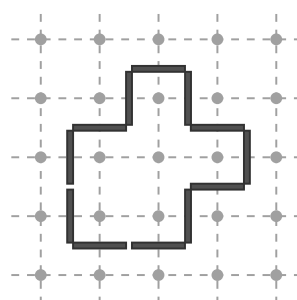

(d)

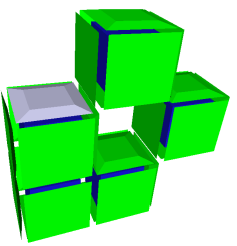

(h)

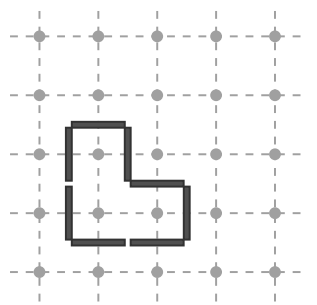

(e)

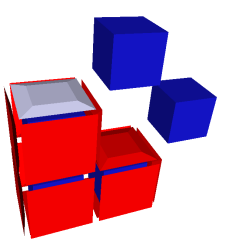

(i)

Figure 4: Illustration of the extraction of the set of boundary surfel (b) from the pixel set (a). The bel adjacency (interior in red, and exterior in green) is illustrated starting from the initial surfel in blue (c). Images (d) and (e) illustrate the interior and exterior tracking from the initial blue surfel. The source codes used to generate these illustrations is given in the directory IllustrationsArticleIPOL of the FrechetAndConnectedCompDemo archive (needs DGtal dependencies with QGLviewer).
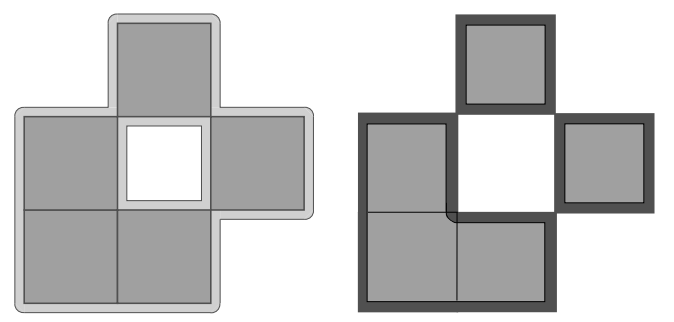

Figure 5: Interpreting digital adjacencies as offsets to the set of cells: (left) outward $\epsilon$-offset corresponding to the exterior adjacency (in light grey), (right) inward $\epsilon$-offset corresponding to the interior adjacency (in dark grey).

or closed surface (with or without boundary). If we know that the surface is closed, the faster variant trackClosedBoundary performs the scan by following only direct adjacent surfels.

For the specific aim of extracting an ordered sequence of boundary elements in $2 \mathrm{D}$, we use a variant of Algorithm 2, track2DBoundary which constructs the ordered sequence from one direction and takes into account the case of open contour by reversing the sequence and by continuing in the other direction.

\subsection{Overall Description}

The main steps of the surface extraction algorithm are the following:

1. $B \leftarrow$ detect boundary surfels (Algorithm 1, method Surfaces: :detectBoundarySurfels in DGtal framework)

2. pick a surfel in $B$

3. $S \leftarrow$ track the boundary component that contains $B$ (Algorithm 2, method Surfaces : : trackBoundary in DGtal framework) 


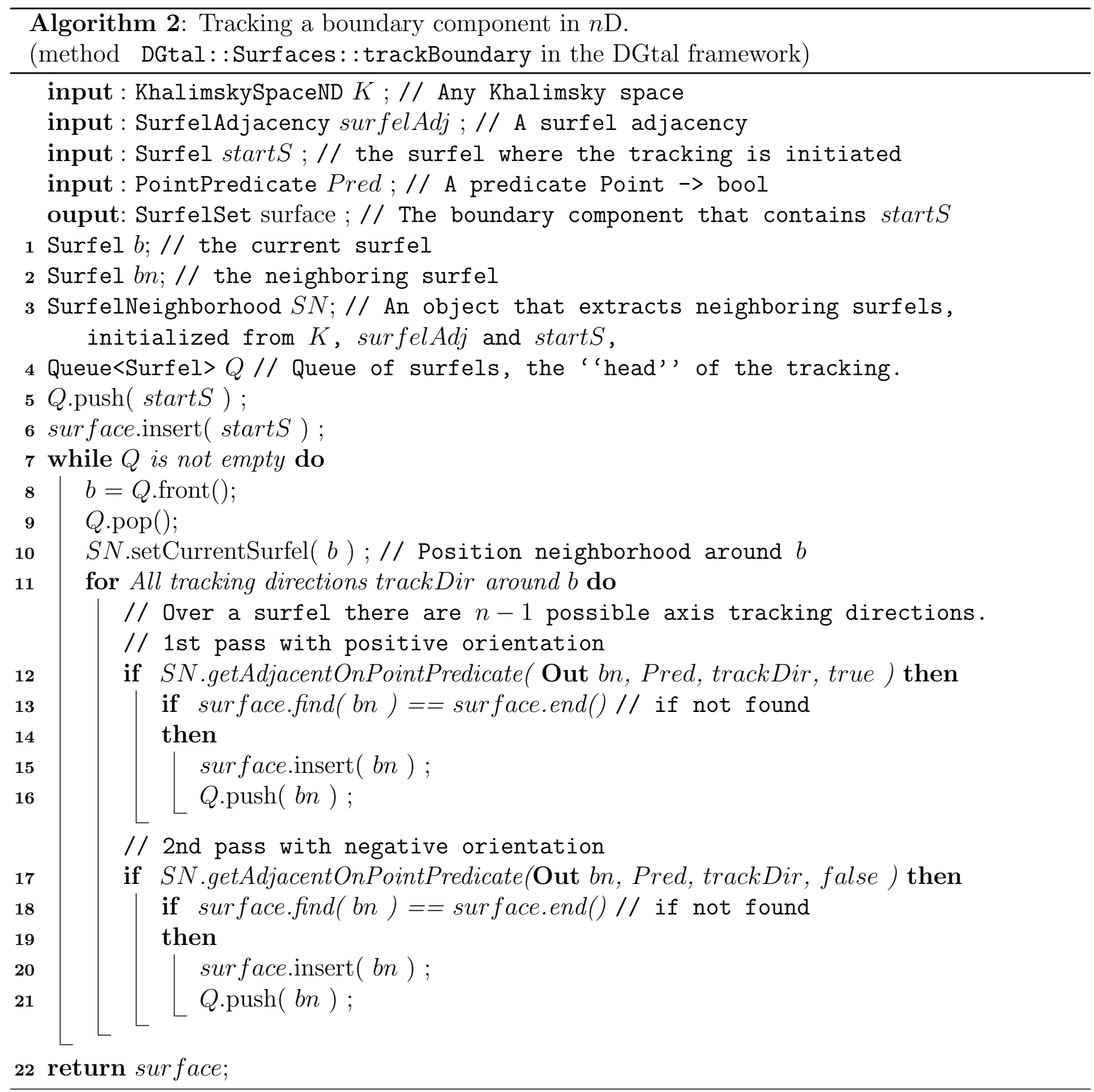

4. remove $S$ from $B$ (line 10-13 of Algorithm 3).

5. go back to 2 until $B$ is empty.

These steps are gathered in Algorithm 3, which extracts for each connected component its set of surfels. In the DGtal library the algorithm is given in the method extractAllConnectedSCell included in class Surfaces (file DGtal/topology/helpers/Surfaces.h). This algorithm can process digital objects given in $n \mathrm{D}$, and the resulting sets of surfels are not given in a particular order. To obtain specifically a set of 2D contours (which can be represented as sequences), we just adapt the algorithm by modifying the tracking (line 8 of Algorithm 3) with the specific 2D tracking (variant of Algorithm 2, method Surfaces : track2DBoundary in the DGtal library). Such variant of Algorithm 3 is available in DGtal with the method DGtal: :Surfaces: : extractAll2DSCellContours. 


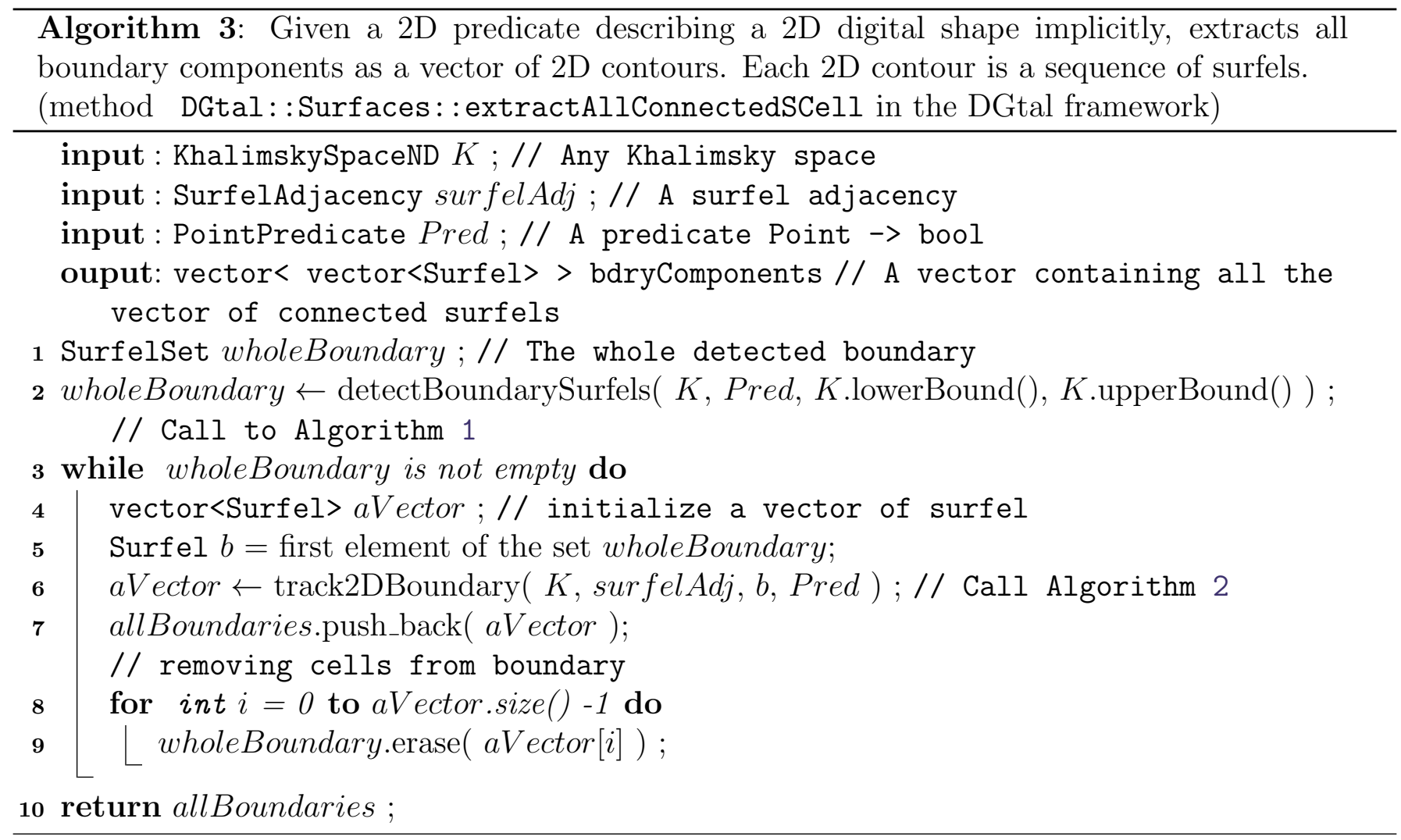

\section{Examples of Applications}

We present two applications of these algorithms, first for 2D contour extraction, and second for 3D connected components extraction.

\subsection{Contour Extraction on 2D Grayscale Images}

The algorithm of contour extraction was applied on the grayscale images of Figure 6 to extract level-set contours. The two adjacency definitions were tested (images (b and $c)$ ), and several thresholds and filtering were used to extract the digital contours in $(\mathrm{d}, \mathrm{e})$. Figure 7 presents complementary results to measure the robustness to noise. These results were obtained from the console command pgm2freeman (from directory FrechetAndConnectedCompDemo/demoIPOL_ExtrConnectedReg). Note that the ordered set of surfels was transformed into a set of pointels by calling

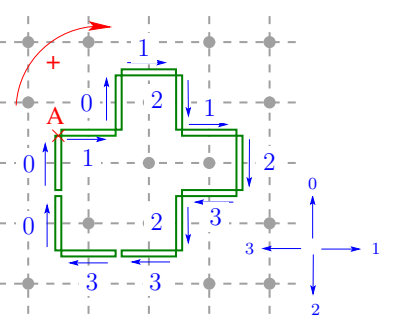
method extractAllPointContours4C.

The obtained contours are represented with a freeman chaincode: it is a word whose letters are codes defining the direction when going from a point to another ( 0 is right, 1 up, 2 left, 3 down). Such a contour is illustrated on the previous floating figure with the starting point $A$ and with the chain code 101212323300. All these experiments can be reproduced from the demonstration source by using the command lines that follows (from the build directory).

- Extraction of contours with different adjacency definitions. (results given on Figure 6 (a-c) with specific colors to highlight each contour.) 


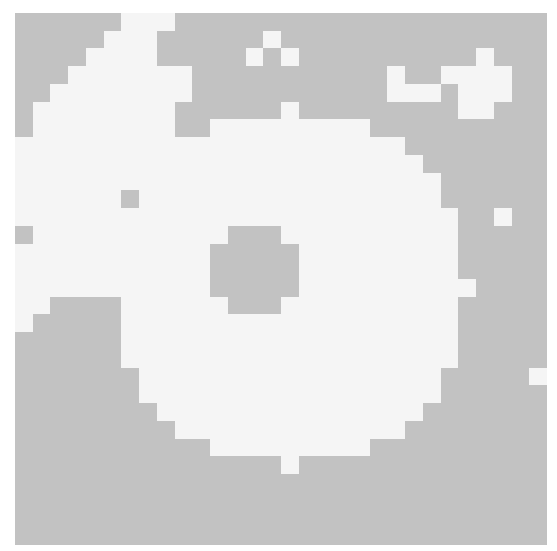

(a) source image.

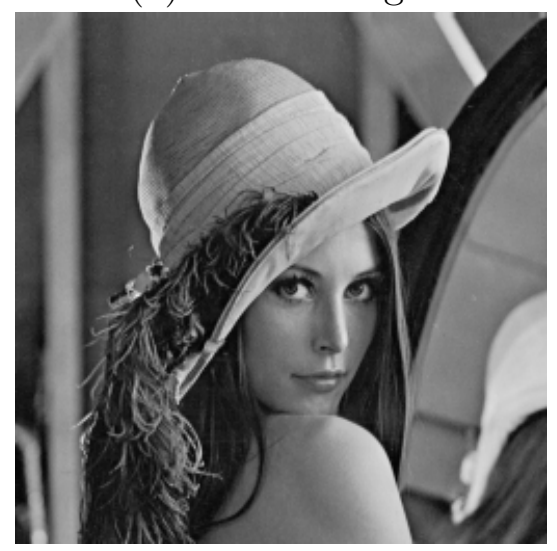

(d) source image.

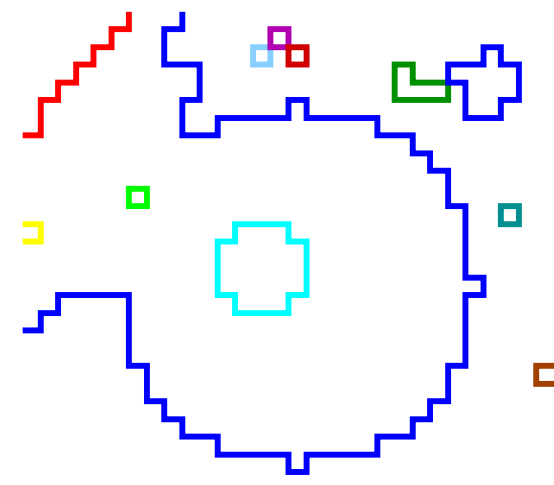

(b) interior adjacency.

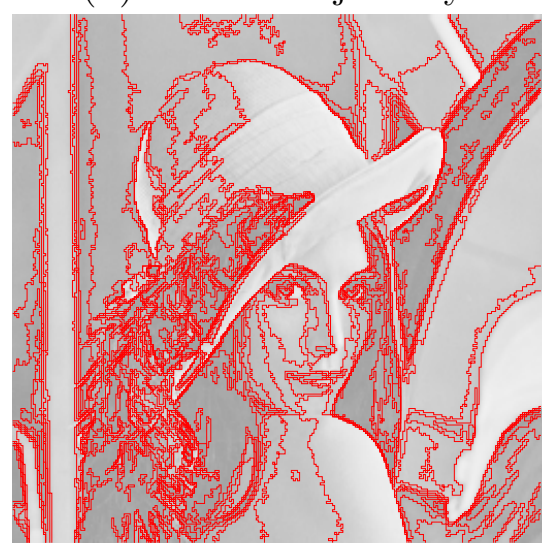

(e) contour extraction from gray scale range.

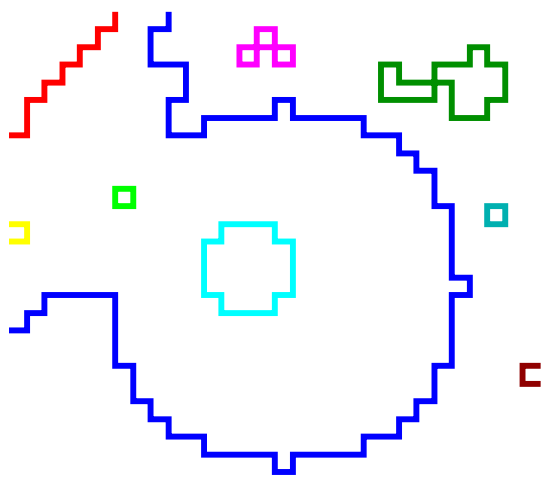

(c) exterior adjacency.

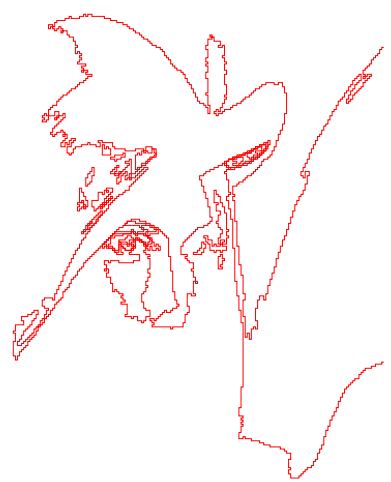

(f) extraction from gray scale range and contour selection.

Figure 6: Visualization of the level set contours extracted with the proposed algorithm (given from pgm2freeman tool). Images (a-c) illustrates the setting of the interior or exterior adjacency parameter. Image (e) shows the extraction of contours from the Lena image with a threshold step equal to 20 and with minimal contour size equal to 20. Image (f) shows a filtering of such contours from a reference point and a minimal distance (respectively equal to $(150,150)$ and 50$)$.

\footnotetext{
shapeTestInt.fc

./demoIPOL_ExtrConnectedReg/pgm2freeman -image . ./demoIPOL_ExtrConnectedReg /Images/shapeTest.pgm -minThreshold $200 \quad$-maxThreshold 255 -badj $1>\hookleftarrow$ shapeTestExt.fc
}

The set of contours can be displayed with the source image in background:

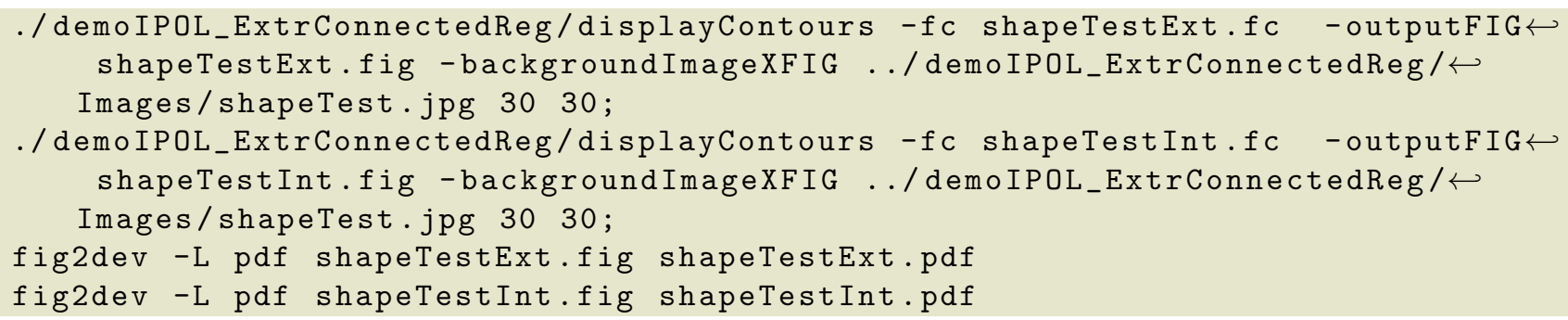

- Extraction of contours given by a threshold range. Extraction of all digital contours from 0 to 256 by step of 20 gray levels with a minimal size equal to 20 (result given in Figure 6 (d,e)): 


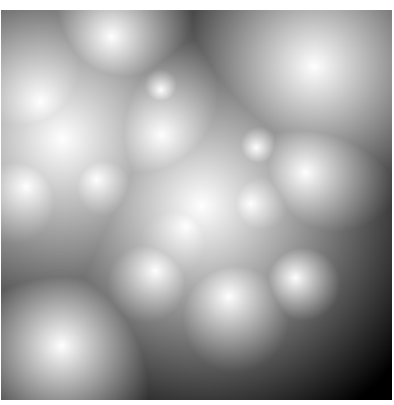

(a) source image.

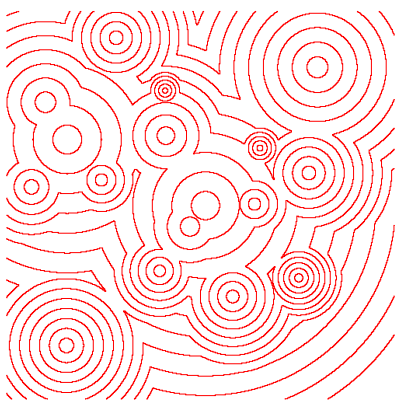

(b) level set contours.

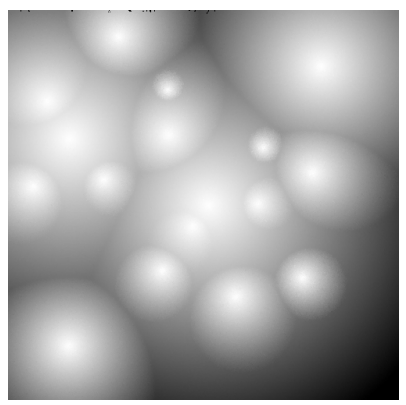

(c) noisy version of (a).

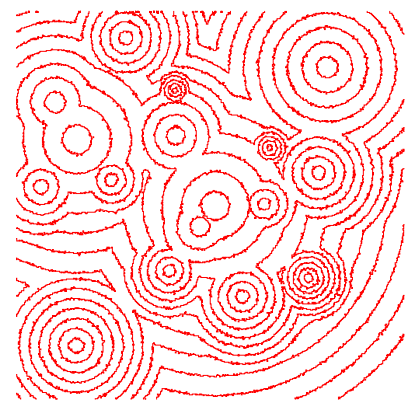

(d) level set contours.

Figure 7: Level set contours extraction on gradient image with (c,d) and without noise (a,b).

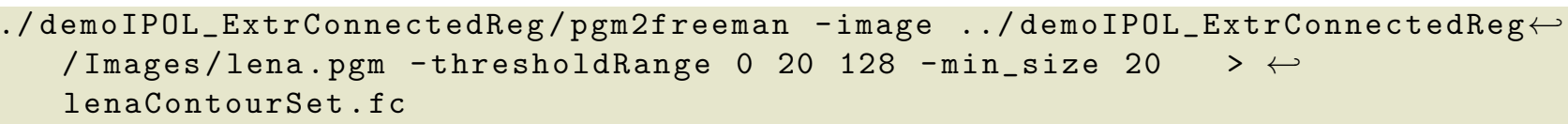

The set of contours can be displayed with the source image in background:

\section{. /demoIPOL_ExtrConnectedReg/displayContours -fc lenaContourSet.fc $-\hookleftarrow$ outputFIG lenaContourSet.fig -backgroundImageXFIG . ./ demoIPOL_ExtrConnectedReg/Images/lenaBG.gif 256256}

- Selection of a set contours from a reference point. (result given in Figure $6(\mathrm{~d}, \mathrm{f})$ )

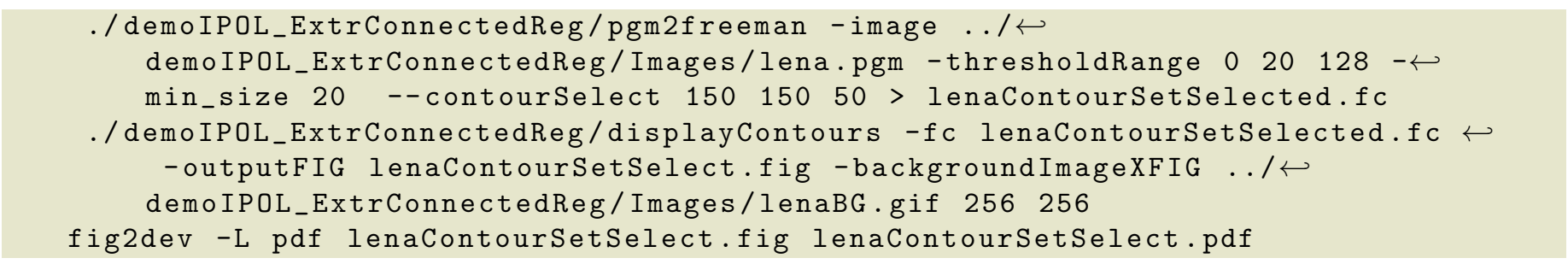

The experiments of Figure 7 are obtained with the same command lines (with images circularGradient.png and circularGradientNoise.png).

\subsection{Surface Tracking on Digital 3D Objects}

We also illustrate the surface extraction for digital 3D objects. First, a simple set of 3D voxels was generated on Figure 8 to display the connected components obtained with different values of adjacency. Each color represents one connected component. As expected, the exterior adjacency connects components which were disconnected with the interior adjacency. Figure 9 shows other surface extraction (with interior adjacency) from a set of voxels obtained by thresholding image (a).

These experiments can be obtained from the demonstration source with the following command that extracts a set of surfels with interior adjacency and export it in a 3D mesh representation (.off format):

\footnotetext{
. /demoIPOL_ExtrConnectedReg/extract3D -image . ./demoIPOL_ExtrConnectedReg/ Images/sample3D1.vol -badj 0 -output surfelCAdjInt.off -exportSRC surfelCSRC. off
}

The threshold can also be set to particular values as for the lobster 3D image of Figure 9: 


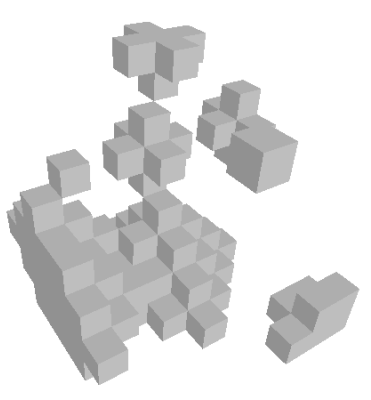

(a) voxel set
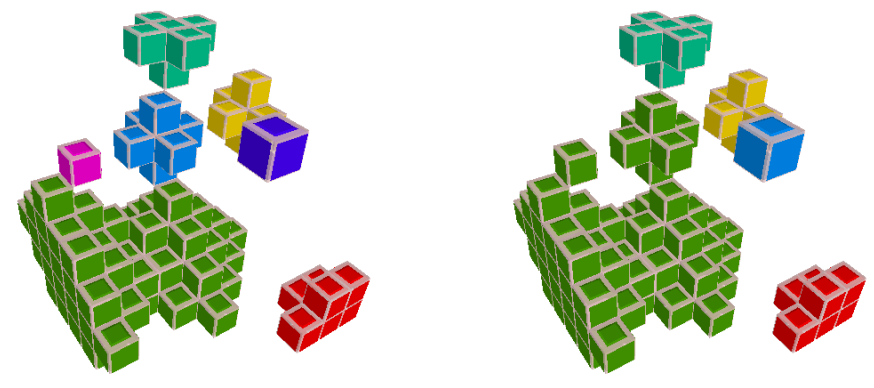

(b) extraction with interior(c) extraction with exterior adjacency adjacency

Figure 8: Illustration of surface tracking on a digital 3D object. Each color represents a connected component. For image (b) (resp. (c)) the connected components are obtained with interior (resp. exterior) adjacency.

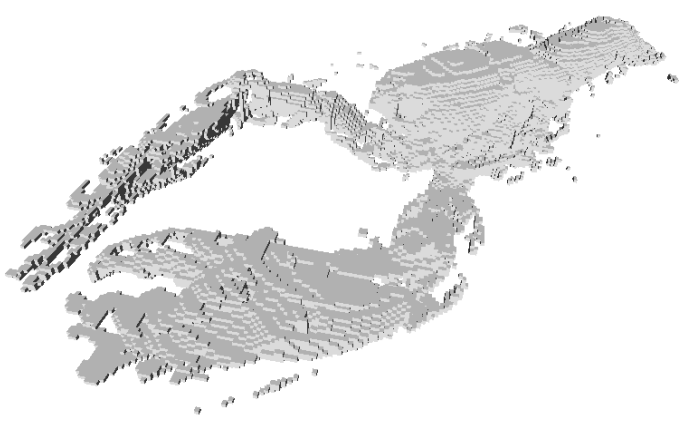

(a) set of voxels.

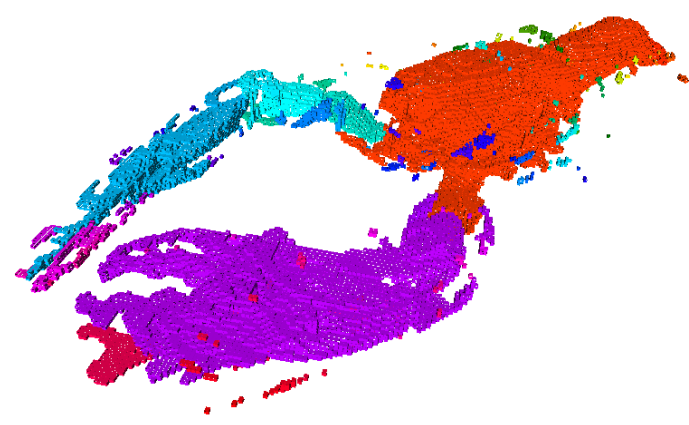

(b) connected set of connected surfels.

Figure 9: Illustration of connected surfel extraction (b) from a set of voxel (a). Each color represents a connected component defined according to the interior adjacency.

\footnotetext{
. / demoIPOL_ExtrConnectedReg/extract3D -image . ./demoIPOL_ExtrConnectedReg/ Images/lobster.vol -badj 0 -threshold 120255 -output lobIntAdj.off $-\hookleftarrow$ exportSRC lobSRC.off
} 


\section{Minimal Code to Include the Contour Extraction in a $\mathrm{C}++$ Program}

To extract the contour directly in your own $\mathrm{C}++$ program you have just to use the following code:

- Include the following header files :

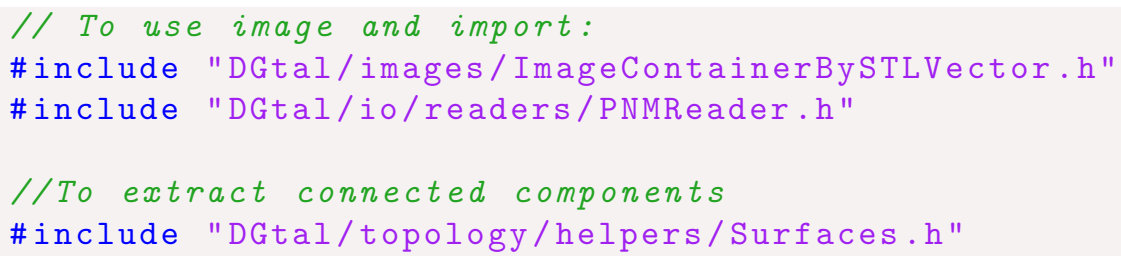

- Import an image:

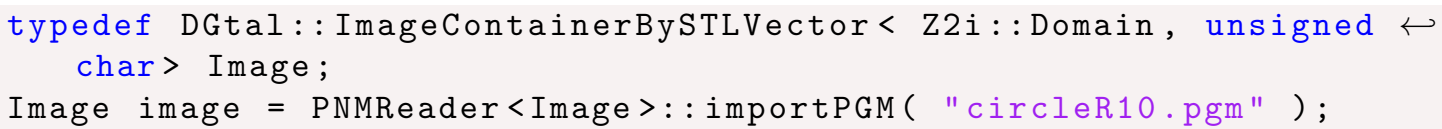

- Define the threshold with Binarizer object:

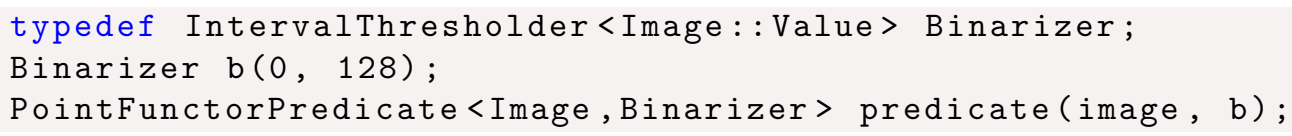

- Extract all contours:

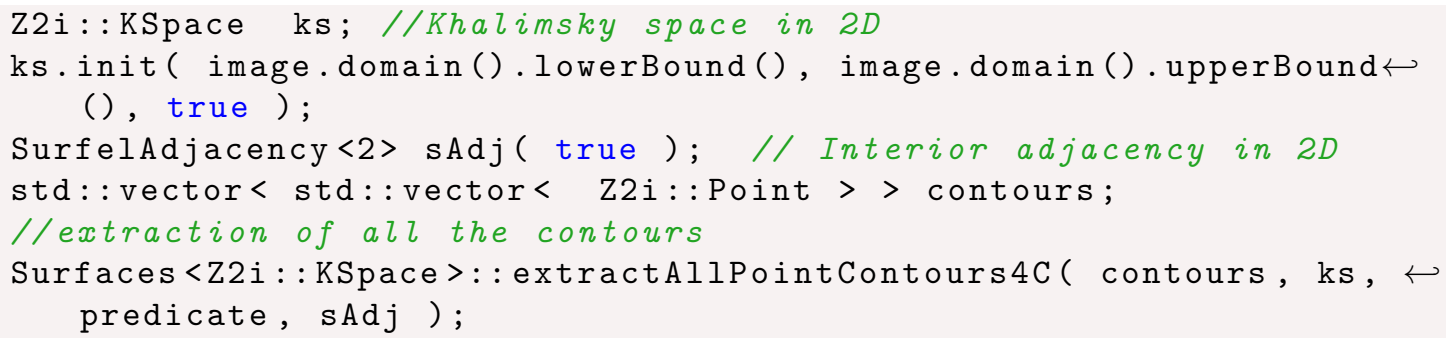

The extraction in 3D images can also be processed in a similar way:

- Include the supplementary header files for the 3D images:

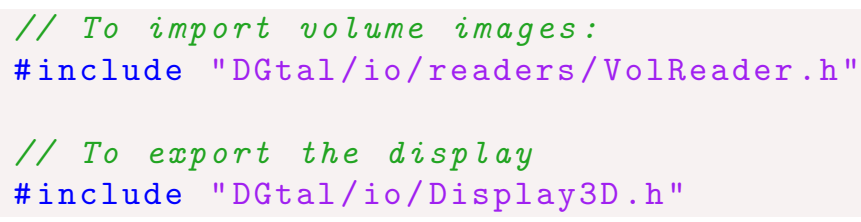


- Import a 3D volume image and define a binarizer:

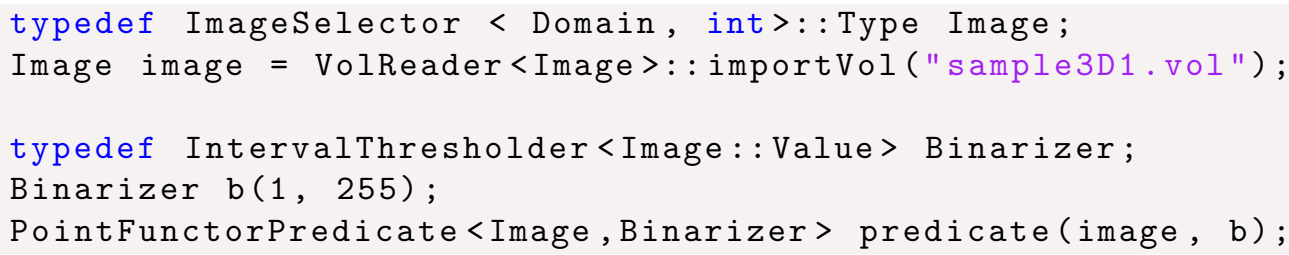

- Extract all the sets of connected surfels:

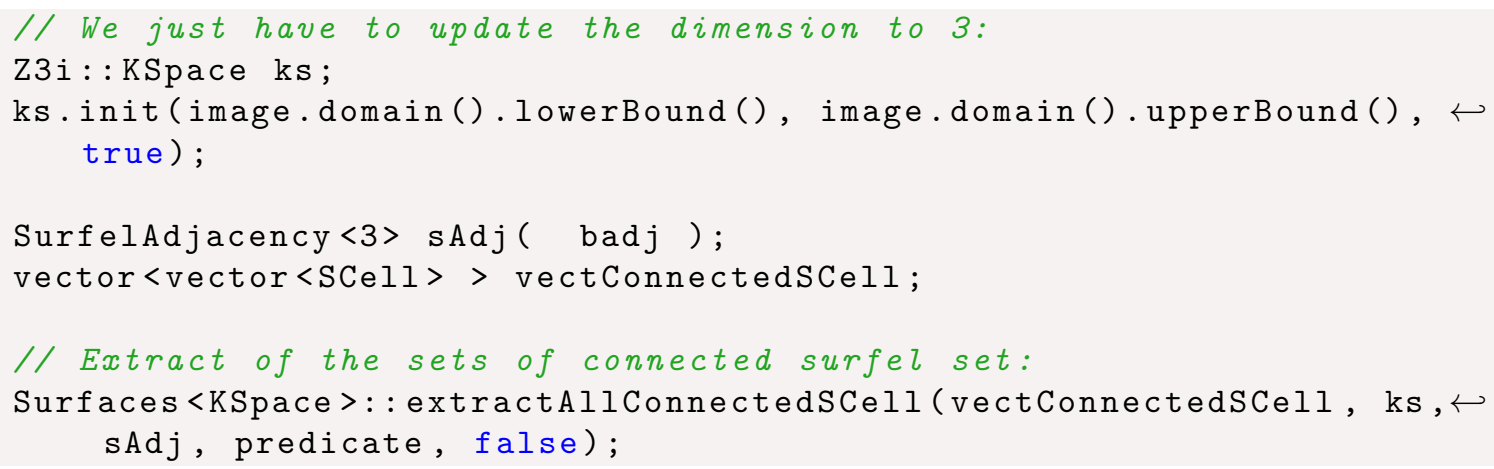

\section{Image Credits}

All images created by the authors except:

VolVis distribution of SUNY Stony Brook, NY, USA. ${ }^{7}$

Standard test image.

\section{References}

[1] G. T. Herman, Discrete multidimensional Jordan surfaces, CVGIP: Graphical Models and Image Processing, 54 (1992), pp. 507-515. http://dx.doi.org/10.1016/1049-9652(92)90070-E.

[2] T. Y. Kong And A. Rosenfeld, Digital topology: Introduction and survey, Computer Vision, Graphics, and Image Processing, 48 (1989), pp. 357-393. http://dx.doi.org/10.1016/ 0734-189X (89) 90147-3.

[3] V. A. Kovalevsky, Finite topology as applied to image analysis, Computer Vision, Graphics, and Image Processing, 46 (1989), pp. 141-161. http://dx.doi.org/10.1016/0734-189x(89) 90165-5.

[4] J.-O. Lachaud, Coding cells of digital spaces: a framework to write generic digital topology algorithms, in Proceedings of 9th International Workshop on Combinatorial Image Analysis (IWCIA'2003), Palermo, Italy, vol. 12 of ENDM, Elsevier, 2003, pp. 337-348. http: //dx.doi.org/10.1016/S1571-0653(04)00497-4.

\footnotetext{
${ }^{7}$ http://labs.cs.sunysb.edu/labs/vislab/volvis-gallery
} 
[5] A. Rosenfeld, Connectivity in digital pictures, Journal of the ACM, 17 (1970), pp. 146-160. http://dx.doi.org/10.1145/321556.321570.

[6] _ Adjacency in digital pictures, Information and Control, 26 (1974), pp. 24-33. http://dx. doi .org/10.1016/s0019-9958(74)90696-2.

[7] J. K. UdupA, Multidimensional digital boundaries, CVGIP: Graphical Models and Image Processing, 56 (1994), pp. 311-323. http://dx.doi.org/10.1006/cgip.1994.1028. 